\title{
Exact results for two-color QCD at low and high density*
}

\section{Takuya Kanazawa}

Department of Physics, The University of Tokyo, Tokyo 113-0033, Japan

E-mail: tkanazawa@nt.phys.s.u-tokyo.ac.jp

\section{Tilo Wettig}

Department of Physics, University of Regensburg, 93040 Regensburg, Germany

E-mail: tilo.wettig@physik.uni-regensburg.de

\section{Naoki Yamamoto}

Institute for Nuclear Theory, University of Washington, Seattle, WA 98195-1550, USA

E-mail: nyama@phys.washington.edu

\begin{abstract}
We discuss a random matrix theory that was originally constructed to describe two-color QCD at low density in the phase with a nonzero chiral condensate. With a particular choice of a parameter, the same random matrix theory also describes the high-density phase of two-color QCD. In this phase a BCS superfluid of diquark pairs is formed, and the pattern of chiral symmetry breaking is very different from that at low density. Analytical results for the spectral density obtained from this random matrix theory allow for the extraction of the BCS gap from lattice data.
\end{abstract}

The XXVIII International Symposium on Lattice Field Theory, Lattice2010

June 14-19, 2010

Villasimius, Italy

${ }^{*}$ Supported by the German Research Foundation (DFG) and by JSPS.

† Speaker. 


\section{Introduction}

Lattice studies of QCD at nonzero quark chemical potential $\mu$ are hindered by the infamous sign problem, see [1] for a review. Two-color QCD with an even number of pairwise degenerate quarks does not have a sign problem and can therefore be simulated on the lattice [2]. It shares many qualitative features, such as confinement and chiral symmetry breaking, with three-color QCD, but the detailed features of both theories are rather different, such as the pattern of chiral symmetry breaking, the particle spectrum, or the phase diagram. Nevertheless, two-color QCD is an interesting theory in its own right. It has been studied in great detail at zero and low density, see, e.g., [3]. In this contribution, we also address the region of high density in which the pattern of chiral symmetry breaking is different from that at low density and in which a BCS superfluid of diquark pairs is expected to be formed because there is an attractive channel between quarks near the Fermi surface. In earlier work [4], we have derived the low-energy effective chiral Lagrangian for $\mu \gg \Lambda_{\mathrm{SU}(2)}$, identified the corresponding $\varepsilon$-regime, and derived Leutwyler-Smilga-type sum rules for the eigenvalues of the Dirac operator. This work has been summarized at Lattice 2009 [5].

In the lowest order of the $\varepsilon$-regime, sometimes also called "microscopic domain", the theory becomes zero-dimensional. This zero-dimensional limit of the theory can alternatively be described by a random matrix theory (RMT). Many examples of such an exact mapping are known, in particular for two- and three-color QCD at zero and low density, see [6, 7] for reviews. Therefore the natural question is what random matrix theory describes the microscopic domain of two-color QCD at high density. The answer to this question was given in [8] and will be reviewed in Sec. 2. The advantage of having a random matrix theory is that it allows us to compute a large number of analytical results characterizing the Dirac eigenvalues, see Sec. 3. This task would be much more difficult in the effective theory. The analytical results at high density contain the BCS gap $\Delta$, which was computed for asymptotically high density in a weak-coupling approach in $[9,10]$, as a parameter. Therefore, $\Delta$ can be extracted from lattice data for the Dirac eigenvalues by matching them to the analytical results from random matrix theory. Another interesting feature of two-color QCD is that it allows us to study the sign problem, either for an odd number $N_{f}$ of flavors by turning on $\mu$, or for even $N_{f}$ by detuning the quark masses from their degenerate values, see Sec. 4.

\section{Random matrix theory at low and high density}

The pertinent random matrix theory for two-color QCD at low density has been formulated in [11], with partition function

$$
Z_{\mathrm{RMT}}(\mu)=\int d P d Q e^{-\frac{1}{2} \operatorname{tr}\left(P P^{T}+Q Q^{T}\right)} \prod_{f=1}^{N_{f}} \operatorname{det}\left(\begin{array}{cc}
m_{f} & P+\mu Q \\
-P^{T}+\mu Q^{T} & m_{f}
\end{array}\right),
$$

where the $m_{f}$ are the quark masses, $P$ and $Q$ are real matrices of dimension $N \times(N+v), d P$ and $d Q$ are Cartesian integration measures, $N$ is assumed to be proportional to the Euclidean spacetime volume $V_{4}$, and $v$ can be identified with the topological charge. Note that sometimes other conventions for the width of the Gaussian distribution of $P$ and $Q$ are used in the literature. The RMT Dirac operator $D(\mu)$ is the matrix in (2.1) with the mass set to zero. 
It was shown in [8] that in the $N \rightarrow \infty$ limit this RMT partition function is identical to the partition function obtained from the static (or zero-dimensional) effective Lagrangian for two-color QCD at low density ${ }^{1}$ given in [3]. More precisely, the two partition functions have the same dependence on the quark masses and on the chemical potential. The mapping between dimensionless RMT quantities and physical quantities is given by

$$
\begin{aligned}
\sqrt{N} m & =m_{\mathrm{phys}} G V_{4}=m_{\pi}^{2} F^{2} V_{4}, \\
\frac{1}{2} N \mu^{2} & =\mu_{\mathrm{phys}}^{2} F^{2} V_{4},
\end{aligned}
$$

where $G$ and $F$ are low-energy constants in the effective Lagrangian in the notation of [3].

Both the random matrix theory (2.1) and the corresponding effective Lagrangian explicitly depend on the chemical potential $\mu$. In contrast, the effective Lagrangian at high density derived in [4] does not explicitly depend on $\mu$. It only depends on the quark masses, which appear in the combination $m^{2} \Delta^{2} V_{4}$. A hint as to what the correct random matrix theory at high density should be can be obtained by noting that (2.1) is basically symmetric under $\mu \rightarrow 1 / \mu$ (except that real and imaginary parts are interchanged). Maximum non-Hermiticity, which is expected at high density, corresponds to $\mu=1$. We therefore conjecture that at high density the random matrix theory is, after a redefinition of the random matrices, given by

$$
Z_{\mathrm{RMT}}=\int d A d B e^{-\frac{1}{4} \operatorname{tr}\left(A A^{T}+B B^{T}\right)} \prod_{f=1}^{N_{f}} \operatorname{det}\left(\begin{array}{cc}
m_{f} & A \\
B^{T} & m_{f}
\end{array}\right),
$$

where the dimension of $A$ and $B$ is again $N \times(N+v){ }^{2}$ In the high-density phase, we restrict ourselves to an even number of flavors.

Let us first check that we obtain the correct pattern of chiral symmetry breaking. To this end, we rewrite the $N_{f}$-flavor determinant resulting from (2.3) in the chiral limit in the form

$$
\operatorname{det}^{N_{f}}\left(\begin{array}{cc}
0 & A \\
B^{T} & 0
\end{array}\right)=\operatorname{det}^{N_{f} / 2}\left(\begin{array}{cc}
0 & A \\
-A^{T} & 0
\end{array}\right) \operatorname{det}^{N_{f} / 2}\left(\begin{array}{cc}
0 & B \\
-B^{T} & 0
\end{array}\right) .
$$

The matrices in the two factors on the RHS of this equation have the form of the chiral orthogonal ensemble of random matrix theory. It was shown in [12] that the symmetry breaking pattern in that ensemble with $N_{f} / 2$ flavors is $\mathrm{U}\left(N_{f}\right) \rightarrow \mathrm{Sp}\left(N_{f}\right)$. Since we have two such factors, (2.3) with $N_{f}$ flavors has the symmetry breaking pattern $\mathrm{U}\left(N_{f}\right) \times \mathrm{U}\left(N_{f}\right) \rightarrow \operatorname{Sp}\left(N_{f}\right) \times \operatorname{Sp}\left(N_{f}\right)$. This agrees with the symmetry breaking pattern in the effective theory due to the formation of a diquark condensate, which is given by $\mathrm{SU}\left(N_{f}\right)_{L} \times \mathrm{SU}\left(N_{f}\right)_{R} \times \mathrm{U}(1)_{B} \times \mathrm{U}(1)_{A} \rightarrow \mathrm{Sp}\left(N_{f}\right)_{L} \times \mathrm{Sp}\left(N_{f}\right)_{R}$ [4].

We have also shown [8] that in the $N \rightarrow \infty$ limit the RMT partition function (2.3) is identical to the partition function of the high-density effective theory in the zero-dimensional limit, i.e., the two partition functions have the same mass dependence. The mapping between the dimensionless RMT mass and the physical mass is now quite different from (2.2a) and given by

$$
m=\frac{\sqrt{3}}{\pi} m_{\text {phys }} \Delta \sqrt{V_{4}} .
$$

\footnotetext{
${ }^{1}$ By low density we here mean the regime of weak non-Hermiticity, see Sec. 3 for the definition of this regime.

${ }^{2}$ Only the case $v=0$ is physically relevant since topology is strongly suppressed at high density.
} 
The arguments presented so far, while giving overwhelming evidence in favor of the equivalence of the random matrix theory (2.3) and the effective theory at high density, do not constitute a full proof. For such a proof one would have to show that all spectral correlation functions are identical in both theories, which requires studying the partially quenched version of the theory. Such a study has not been done yet, but we have no doubt that the outcome would be positive.

\section{Exact results from random matrix theory}

We can now proceed to compute spectral correlation functions from the random matrix theory in the $N \rightarrow \infty$ limit. At $\mu=0$ the RMT eigenvalues $\lambda$ are purely imaginary, while at $\mu \neq 0$ they are either purely real, purely imaginary, or come in complex conjugate pairs. We are mainly interested in the so-called microscopic spectral density of the small eigenvalues, i.e., we rescale all eigenvalues by a quantity $\delta$ that is, up to a numerical prefactor, equal to the mean level spacing near zero. This results in complex numbers $z=\lambda / \delta$ of order $O(1)$. To see an effect of the quark masses on the small eigenvalues we need to rescale them in the same way, resulting in $\hat{m}_{f}=m_{f} / \delta$.

The random matrix theory can be solved in two different regimes:

- In the regime of weak non-Hermiticity, the combination $\hat{\mu}^{2}=2 N \mu^{2}=4 \mu_{\mathrm{phys}}^{2} F^{2} V_{4}$ is kept fixed in the limit $N \rightarrow \infty$. While this regime might appear to be mainly of academic interest since $\mu \rightarrow 0$ in the thermodynamic limit, it has an important phenomenological application, i.e., the extraction of the low-energy constants $G$ and $F$ from lattice data. In our conventions we have $\delta=1 / 2 \sqrt{N}$ in this regime, i.e., $z=2 \sqrt{N} \lambda$ and $\hat{m}=2 \sqrt{N} m$.

- In the regime of strong non-Hermiticity, $\mu$ is kept nonzero in the limit $N \rightarrow \infty$. The analytical results in this regime are the $\hat{\mu} \rightarrow \infty$ limits of the corresponding weak non-Hermiticity results. Their functional form is identical for all $0<\mu \leq 1$, and the $\mu$-dependence only enters through a rescaling of the eigenvalues. In our conventions we have $\delta=1$ in this regime so that no $N$-dependent rescaling of the eigenvalues and the masses is necessary.

In [13] analytical results for the microscopic spectral "density" (which we put in quotation marks since this quantity can become negative if there is a sign problem) were obtained in both of the above-mentioned regimes in the quenched case, i.e., for $N_{f}=0$ flavors. In the meantime, the generalization to the unquenched case has been worked out [14]. Since the analytical results are rather cumbersome we will not present them here. Important features of the results are exhibited in Figs. 1 through 4. Comments on these features are given in the figure captions.

\section{The sign problem}

As a good measure of the sign problem in two-color QCD we define the quantity

$$
\langle\operatorname{sgn} \operatorname{det}(D+m)\rangle_{\| N_{f}||} \equiv \frac{\left\langle\operatorname{sgn} \operatorname{det}(D+m) \prod_{f=1}^{N_{f}}\left|\operatorname{det}\left(D+m_{f}\right)\right|\right\rangle_{N_{f}=0}}{\left\langle\prod_{f=1}^{N_{f}}\left|\operatorname{det}\left(D+m_{f}\right)\right|\right\rangle_{N_{f}=0}},
$$

see [14] for a more detailed discussion. 

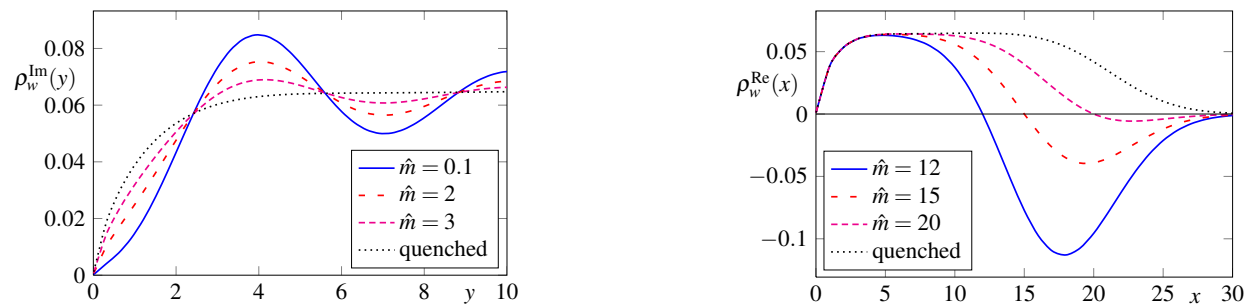

Figure 1: Microscopic spectral "density" of the purely imaginary (left) and purely real (right) eigenvalues in the regime of weak non-Hermiticity for $N_{f}=1, \hat{\mu}=3, v=0$, and different values of $\hat{m}$. The density of the real eigenvalues goes through zero for $x=\hat{m}_{f}$.
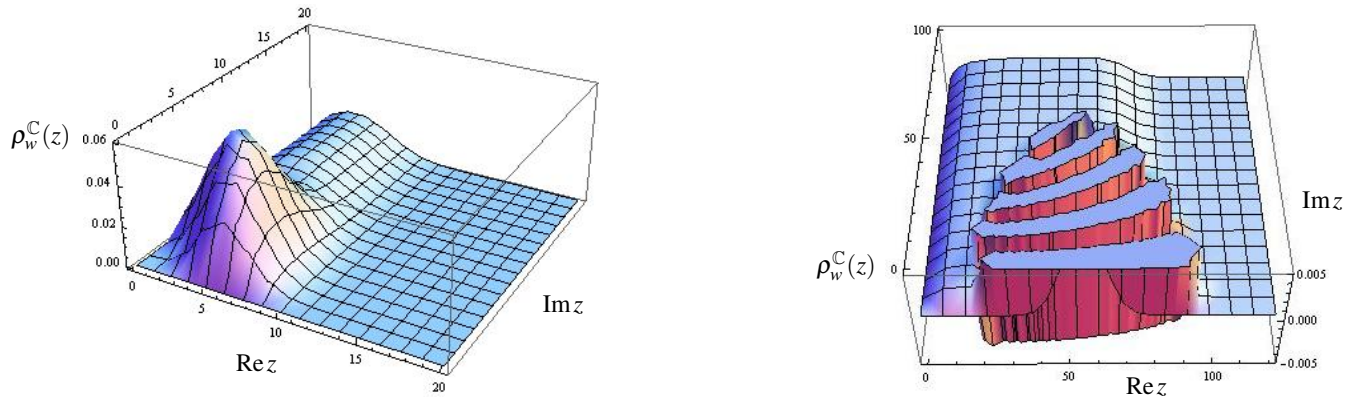

Figure 2: Microscopic spectral "density" of the complex eigenvalues in the regime of weak non-Hermiticity for $N_{f}=1$ and $v=0$. Left: $\hat{\mu}=1.8$ and $\hat{m}=0$. The massless quark causes a depletion of the density near the origin. Right: $\hat{\mu}=6$ and $\hat{m}=20$. For large $\hat{\mu}$ there is an elliptical domain in which the "density" oscillates strongly.
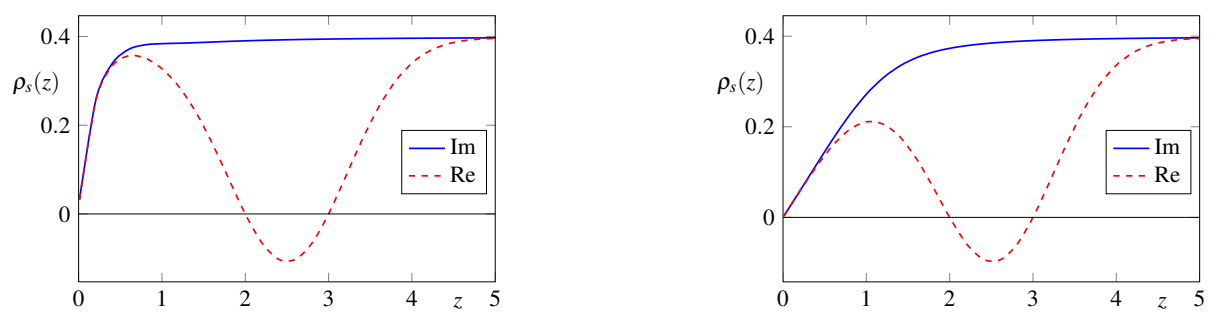

Figure 3: Microscopic spectral "density" of the purely imaginary (solid) and purely real (dashed) eigenvalues in the regime of strong non-Hermiticity for $N_{f}=2, \hat{m}_{1}=2, \hat{m}_{2}=3, v=0$ (left), and $v=2$ (right). The density of the real eigenvalues goes through zero for $x=\hat{m}_{f}$.
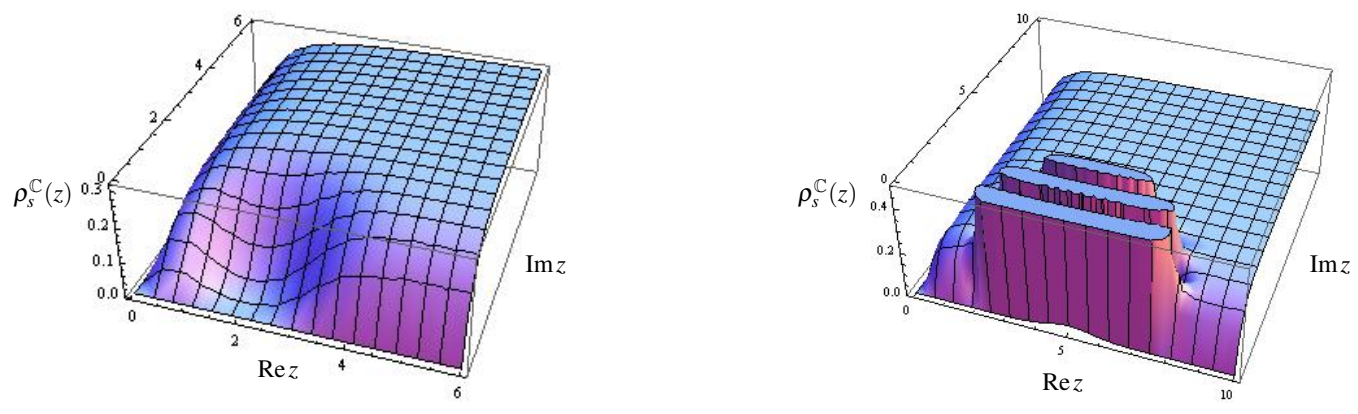

Figure 4: Microscopic spectral "density" of the complex eigenvalues in the regime of strong non-Hermiticity for $N_{f}=2$ and $v=0$. Left: $\hat{m}_{1}=\hat{m}_{2}=2$. Degenerate masses generate a dip in the spectrum at $z=\hat{m}$. Right: $\hat{m}_{1}=2, \hat{m}_{2}=8$. Unequal masses result in a domain of strong oscillations, indicative of the sign problem. 

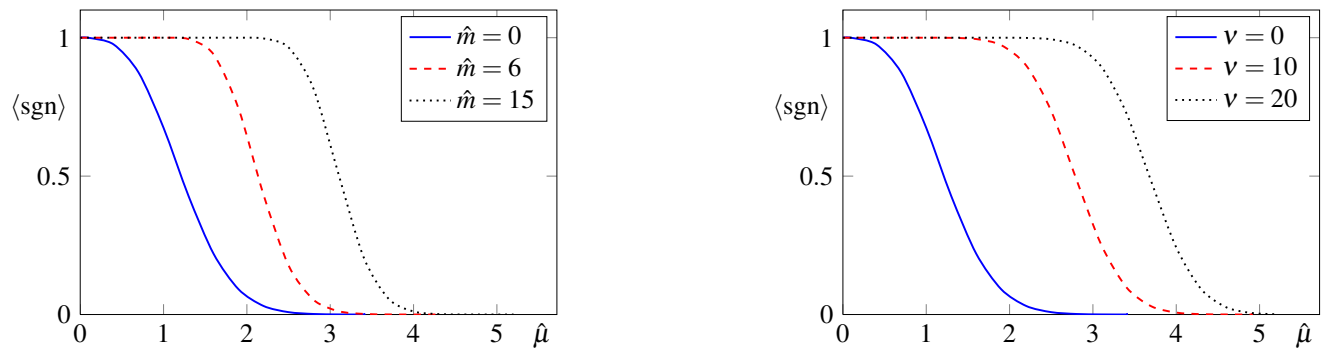

Figure 5: Average sign at weak non-Hermiticity for $N_{f}=1$ as a function of $\hat{\mu}$. Left: $v=0$ is kept fixed and $\hat{m}$ is varied. Right: $\hat{m}=0$ is kept fixed and $v$ is varied.

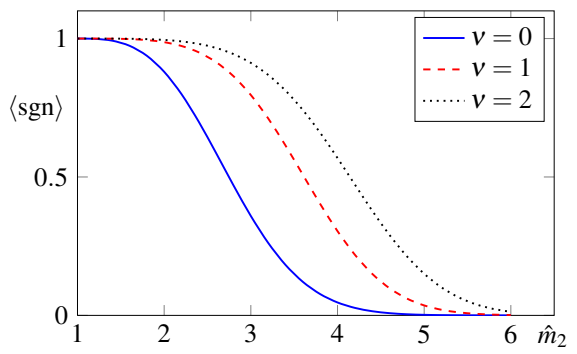

Figure 6: Average sign at strong non-Hermiticity for $N_{f}=2, \hat{m}_{1}=1$, and $v=0,1,2$ as a function of $\hat{m}_{2}$.

We first consider the regime of weak non-Hermiticity. We choose $N_{f}=1$ and turn on $\hat{\mu}$ to study its effect on the average sign, see Fig. 5. It is evident from the plots that the sign problem (i) increases with increasing $\hat{\mu}$, (ii) decreases with increasing $\hat{m}$, and (iii) decreases with increasing $v$ (in agreement with [15]). A quantitative analysis [14] reveals that in the thermodynamic limit the average sign makes a first-order transition from 1 to 0 at $\hat{\mu}=\sqrt{\hat{m} / 2}$, which in physical units corresponds to a critical chemical potential $\mu_{\text {phys }}=m_{\pi} / 2$.

Next we consider the regime of strong non-Hermiticity. We choose $N_{f}=2$ and detune the quark masses. The effect on the average sign is shown in Fig. 6. The sign problem is absent for $\hat{m}_{1}=\hat{m}_{2}$ and increases as $\left|\hat{m}_{1}-\hat{m}_{2}\right|$ increases. It again decreases with increasing $v$.

\section{Conclusions}

We have shown that a single random matrix theory describes two-color QCD at low density in the regime of weak-Hermiticity and at high density in the BCS superfluid phase, depending on the choice of the RMT parameter $\mu$ and on the rescaling factors in (2.2) and (2.5). These two regimes have very different symmetry breaking patterns. It would be interesting to investigate the applicability of random matrix theory in the region of intermediate densities, where intriguing phenomena such as a BEC-BCS crossover have been conjectured [16].

The analytical RMT results can be used to extract physical parameters such as $\Delta$ from lattice data. Two-color lattice simulations with adjoint staggered fermions, which are in the same symmetry class as continuum fundamental fermions [17], are currently underway to test our RMT predictions. 


\section{References}

[1] P. de Forcrand, Simulating QCD at finite density, PoS LAT2009 (2009) 010, [arXiv: 1005.0539 ].

[2] J. B. Kogut, D. K. Sinclair, S. J. Hands, and S. E. Morrison, Two-colour QCD at non-zero quark-number density, Phys. Rev. D64 (2001) 094505, [hep-lat/ 0105026 ].

[3] J. B. Kogut, M. A. Stephanov, D. Toublan, J. J. M. Verbaarschot, and A. Zhitnitsky, QCD-like theories at finite baryon density, Nucl. Phys. B582 (2000) 477-513, [hep-ph/ 0001171$].$

[4] T. Kanazawa, T. Wettig, and N. Yamamoto, Chiral Lagrangian and spectral sum rules for dense twocolor QCD, JHEP 08 (2009) 003, [arXiv: 0906.3579 ].

[5] T. Kanazawa, T. Wettig, and N. Yamamoto, Chiral Lagrangian and spectral sum rules for two-color QCD at high density, PoS LAT2009 (2009) 195, [arXiv: 0910 .2300].

[6] J. J. M. Verbaarschot and T. Wettig, Random matrix theory and chiral symmetry in QCD, Ann. Rev. Nucl. Part. Sci. 50 (2000) 343-410, [hep-ph/ 0003017 ].

[7] G. Akemann, Matrix models and QCD with chemical potential, Int. J. Mod. Phys. A22 (2007) 1077-1122, [hep-th/0701175].

[8] T. Kanazawa, T. Wettig, and N. Yamamoto, Chiral random matrix theory for two-color QCD at high density, Phys. Rev. D81 (2010) 081701, [arXiv:0912.4999].

[9] D. T. Son, Superconductivity by long-range color magnetic interaction in high-density quark matter, Phys. Rev. D59 (1999) 094019, [hep-ph/9812287].

[10] T. Schafer, QCD and the eta' mass: Instantons or confinement?, Phys. Rev. D67 (2003) 074502 , [hep-lat/0211035].

[11] G. Akemann, M. J. Phillips, and H. J. Sommers, Characteristic polynomials in real Ginibre ensembles, J. Phys. A42 (2008) 012001, [arXiv: 0810.1458$].$

[12] A. M. Halasz and J. J. M. Verbaarschot, Effective Lagrangians and chiral random matrix theory, Phys. Rev. D52 (1995) 2563-2573, [hep-th/ 9502096 ].

[13] G. Akemann, M. J. Phillips, and H. J. Sommers, The chiral Gaussian two-matrix ensemble of real asymmetric matrices, J. Phys. A43 (2010) 085211, [arXiv:0911.1276].

[14] G. Akemann, T. Kanazawa, M. Phillips, and T. Wettig, Random matrix theory of unquenched two-color QCD with nonzero chemical potential, (2010) to be published.

[15] J. C. R. Bloch and T. Wettig, Random matrix analysis of the QCD sign problem for general topology, JHEP 03 (2009) 100, [arXiv: 0812 . 032 4].

[16] K. Splittorff, D. T. Son, and M. A. Stephanov, QCD-like Theories at Finite Baryon and Isospin Density, Phys. Rev. D64 (2001) 016003, [hep-ph/ 0012274 ].

[17] S. Hands et. al., Numerical study of dense adjoint matter in two color QCD, Eur. Phys. J. C17 (2000) 285-302, [hep-lat/0006018]. 